\title{
EXTENSION OF RCC TOPOLOGICAL RELATIONS FOR 3D COMPLEX OBJECTS COMPONENTS EXTRACTED FROM 3D LIDAR POINT CLOUDS
}

\author{
Xu-Feng XING ${ }^{\text {a }}$, Mir Abolfazl Mostafavi ${ }^{a, *}$, Chen WANG ${ }^{\text {a }}$ \\ ${ }^{a}$ Dept. of Geomatics, Laval University, Québec, Canada - (xufeng.xing.1, chen.wang.1)@ulaval.ca, Mir- \\ Abolfazl.Mostafavi@scg.ulaval.ca
}

WG III/2

KEY WORDS: Topological relations, planar regions, components, automatic 3D modelling, point cloud

\begin{abstract}
:
Topological relations are fundamental for qualitative description, querying and analysis of a 3D scene. Although topological relations for 2D objects have been extensively studied and implemented in GIS applications, their direct extension to 3D is very challenging and they cannot be directly applied to represent relations between components of complex 3D objects represented by 3D B-Rep models in $R^{3}$. Herein we present an extended Region Connection Calculus (RCC) model to express and formalize topological relations between planar regions for creating 3D model represented by Boundary Representation model in $R^{3}$. We proposed a new dimension extended 9-Intersection model to represent the basic relations among components of a complex object, including disjoint, meet and intersect. The last element in $3 * 3$ matrix records the details of connection through the common parts of two regions and the intersecting line of two planes. Additionally, this model can deal with the case of planar regions with holes. Finally, the geometric information is transformed into a list of strings consisting of topological relations between two planar regions and detailed connection information. The experiments show that the proposed approach helps to identify topological relations of planar segments of point cloud automatically.
\end{abstract}

\section{INTRDUCTION}

Spatial relations include topological, metric and directional relations and together with semantic information are used for describing a scene qualitatively (Mark, 1994). Topological relations between geographical objects are necessary for spatial analysis in GIS. These relations can be queried and analysed independently from geographic coordinate system definition and the specific location of objects. Topological relations describe relative spatial relations with respect to reference objects. Hence topological relations are invariant and do not change with topological transformations, such as translation, scaling, and rotation (Egenhofer, 1990b).

In general, topological relations between spatial objects are derived from Region Connection Calculus (RCC-8) (Egenhofer, 1989;Egenhofer, 1991) in $R^{2}$. Existing RCC have been further applied to Qualitative Spatial Reasoning (QSR) in the field of GIS, robotics, medicine and engineering problems for the reasoning of the topological relationships in $R^{2}$ (Cohn, 2008). Here, a region is defined as a 2-cell with a non-empty, connected interior (Egenhofer, 1990a). Additionally, the 4Intersection Model (4IM) (Egenhofer, 1991), 9-Intersection Model(9IM) (Clementini, 1993) and Dimensionally Extended models (DE) (Clementini, 1993) are widely adopted and implemented for describing topological relations for spatial analysis. Topological relations between spatial objects can be described based on relations defined for $2 \mathrm{D}$ regions in $\mathrm{RCC}$ model. Basic relations between two regions include disjoint, meet, overlap, contain, cover, coveredBy, containedBy and equal (Egenhofer, 1990b;Randell, 1992).
The definitions of topological relations between spatial objects in $R^{3}$ are closely related to $3 \mathrm{D}$ objects models. A $3 \mathrm{D}$ spatial object can be modelled as a solid geometry or represented by its boundaries. Thus, topological relations between spatial objects in $R^{3}$ can be divided into two aspects: topological relations between 3D complex objects and topological relations among components of a complex object. For 3D objects represented by boundary representation (B-Rep), there exists the concepts of the interior, boundary, and exterior of objects. The topological relations between 3D spatial objects represented by B-Rep can be directly extended to define eight basic topological relations in $R^{3}$ (Zlatanova, 2004). However, in this paper, we concentrate on topological relations among object components in a single 3D spatial object represented by B-Rep rather than relations between 3D complex objects. However, the topological relations among objects components are not the same as relations between complex objects themselves. Additionally, the boundaries of 2D objects can be extracted and represented by polygons in $R^{2}$, similarly, the boundaries of 3D objects can be modelled as facets. Here, facets are composed of small planar surfaces such as triangles for representing surfaces. Referring to the definition of the region in $R^{2}$, a planar region in $R^{3}$ is defined as a planar surface area with a non-empty, connected interior in $R^{3}$. A planar region is described by its boundaries and the parameter of the plane equation in which planar regions are located. Therefore, the topological relations between components of a complex object represented by B-Rep can be modelled as the relations between planar regions in $R^{3}$.

For creating 3D B-Rep models from point clouds, we need the topological relations among points, the relations among components of complex objects and objects themselves in three

\footnotetext{
* Corresponding author
} 
different levels (Pigot, 1991). For representing objects with complex structure, such as buildings, using B-Rep models, topological relations among components provide the connection of components to form a whole 3D model with interior space. Unlike the definitions of 3D simple geometric primitives, such as the sphere, cube, and cylinder (Leopold, 2015), a planar region in $R^{3}$ does not have a volume. In 3D B-Rep models, a planar region can represent a part of a 3D complex object boundary. The topological relations between planar regions connect all boundaries parts together to represent a 3D object. Thus, topological relations between planar regions play an important role in creating 3D B-Rep models from a point cloud. More importantly, the definition of the boundary of a planar region in $R^{3}$ (for example a part of a wall represented as a rectangle in $R^{3}$ ) depends on plane equation that contains planar regions and the definition of its boundary is needed to determine the topological relations among planar regions.

The existing topological models have limitations to meet the requirements of building topological relations of object components to form a whole 3D B-Rep model. For example, in Table 1, the overlap relation between region $\mathrm{A}$ and $\mathrm{B}$ in $R^{2}$ can be represented by $4 \mathrm{IM}$ as a matrix $[11 ; 11]$. But if RCC-8 is directly applied to determine topological relations, we will get $[00 ; 10]$ in $R^{3}$. It cannot be defined as overlap. It is needed to extend these relations to describe the topological relation of two planar regions in $R^{3}$

\begin{tabular}{|l|c|c|}
\hline Space type & Figure example & Representation of 4IM \\
\hline$R^{2}$ & A & {$\left[\begin{array}{ll}1 & 1 \\
1 & 1\end{array}\right]$} \\
\hline$R^{3}$ & A & {$\left[\begin{array}{ll}0 & 0 \\
1 & 0\end{array}\right]$} \\
\hline
\end{tabular}

Table 1. The differences of topological relations between two regions in $R^{2}$ and $R^{3}$

Some studies propose RCC extension in response to those limits. For example, topological relations extended from RCC model are defined and distinguished as non-occlusion, partial occlusion and complete occlusion relations in the projected planes in a specific perspective in RCC-3D (Albath, 2010b) and VRCC-3D+ (Albath, 2010b;Sabharwal, 2011). However, they did not involve the topological relations among objects components. Therefore, a formalized representation and discrimination method for the topological relations between 3D planar regions is an indispensable part of 3D modelling and spatial analysis.

In this paper, we are concerned with the determination of the topological relations between planar regions in 3D space generated from point clouds (ex. LiDAR point clouds). We present an overview of generalized topological models for describing relations between planar regions in $R^{3}$ and then based on the definitions of basic eight topological relations, a new extended topological model from RCC is proposed to define topological relations among planar regions. In addition, the validation of the extended RCC model is conducted to identify topological relations between buildings components consisting of planar regions extracted from point clouds.

The reminder of this paper is organized as follows. Section 2 briefly discusses 3D model representation methods that are suitable for 3D modelling from point clouds, and their advantages and limitations. In addition, the RCC, 4IM, 9IM and DE9IM in $R^{2}$, and other studies related to topological relations in $R^{3}$ are discussed. In section 3, topological relations for planar regions are defined and formally represented. Moreover, the steps for deciding topological relations are presented. Section 4 validates the proposed topological models for planar regions on a point cloud dataset. The processes of deciding relations among planar regions segmented from point clouds are given as well. Section 5 outlines conclusions and future work.

\section{RELATED WORK}

\subsection{D Objects Representation Methods}

Boundary Representation, also called as B-Rep, describe 3D objects boundaries composed of vertices, edges and faces (JARROUSH, 2004). In a B-Rep model, geometric information is derived from the coordinate of vertices. The geometric information describes its shapes and its boundaries constrained by vertices, edges, and faces. The topology between different components describes the connectivity relationships among basic primitives of boundaries. For example, a part of point cloud observed to model a planar wall provides basic geometric information through the coordinate points. The shape of a wall is determined by the parameters of a plane equation and its boundary. The connection between several walls are described by topological relations. For objects with complex structure, BRep represents objects based on their fundamental geometric primitives to create a complete model with the help of topology. Therefore, B-Rep is capable of creating 3D models of complex objects, and it can describe their surface boundaries accurately. However, B-Rep model is not very efficient for the representations of complex solid objects because it needs a large volume of data to represent them (Koussa, 2009).

Constructive Solid Geometry (CSG) model create a complex object using Boolean operations (including intersection, union, and difference) among basic primitives, such as cubes, cylinders, cones, and spheres (Foley, 1996). However, it has limitations to create complex objects with irregularly curved surfaces. More importantly, CSG does not provide a unique representation, which will yield different results (Foley, 1996). In conclusion, for automatic 3D modelling of point clouds, the simple operations between simple primitives in CSG is not enough to represent complex structures of objects and irregular shapes of objects.

Another approach for modelling 3D objects is the "Parametric approach" (Koussa, 2009). In this approach, an object is modelled by its primitive components. These primitive geometric objects are defined by a set of parameters. Geometric information of these objects consists of length, height, width, angle and diameter. These geometric parameters and the relationships among components are allowed to be defined by users. Thus, it is flexible to represent geometric models, and some semantic information can be attached. In general, these kinds of information are manually set by users (Koussa, 2009). However, if the geometric parameter can be acquired from point clouds, this method is helpful to represent planar regions. Therefore, this method can be employed in automatic 3D modelling if geometric parameters are obtained from point cloud automatically. For those nonplanar primitives (such as a cylinder, cone, and torus), parametric approach briefly describes geometric models by several parameters as well. However, the topological relations between planar regions are the cores of 3D topologies because complex shapes can be decomposed into 
simple planar primitives. Also, for the purpose of determining topological relationships of planar regions from point clouds, the accurate boundary information still require geometric parameters of planar regions.

For automatic 3D modelling from point clouds, B-Rep is more adaptable because point clouds record the outer surface information of objects in points. As for various kinds of objects, B-Rep has the capability of describing complex shapes and spatial structures of objects due to the topological relationships built from vertices, edges, and faces. Additionally, B-Rep can represent 3D objects with complex structure through the topological relationships among simple geometric primitives. Especially for complex irregular shapes, B-Rep can use triangulations for surface representation where topologies can be defined the relations between simple triangular faces.

In conclusion, considering the advantages and limitations of the presented models, we propose a hybrid approach that combines parametric approach and B-Rep models to define topological relations among components of a complex objects in $3 \mathrm{D}$ space.

\subsection{Models for Topological Relations}

2.2.1 Calculus-based Spatial Logic Model: In 2D space, Region Connection Calculus (RCC) is one of the fundamental methods for the definition of topological relationships. In this model, topological relationships are grouped into six categories including relations between point-point, point-line, pointregion, line-line, line-region, region-region. Among these relations, region-region relations are the most commonly used to express topological relations between different primitives (Deng, 2007). The existing eight topological relations (RCC-8) are (Randell, 1992): disconnected (DC), partial overlap (PO), equal (EQ), externally connected (EC), tangential proper part (TPP), non-tangential proper part (NTPP) and their inverse relations are TPPi and NTPPi respectively. Additionally, RCC describes the logics representations of spatial relations between regions (Randell, 1992). Also, these eight relations can also be converted from one relation to another to describe topological relations in a dynamic scene.

2.2.2 Intersection Model: In “4-Intersection” Model (4IM) (Egenhofer, 1989;Egenhofer, 1991), eight topological relationships between two regions are defined. They are disjoint, meet, overlap, contain, cover, coveredBy, containedBy and equal. These relations correspond to RCC-8 relations: DC, EC, PO, NTPP, TPP, TPPi, NTPPi, EQ, respectively. They are obtained by the intersection between boundaries and interiors of two primitives geometries (ex. region). A matrix $T(A, B)$ consists of the intersection of boundaries and interiors of region $\mathrm{A}$ and $\mathrm{B}$. The intersection values are distinguished only by "empty" and "non-empty" value. 0 and 1 represent the empty and non-empty, respectively.

$$
T(A, B)=\left[\begin{array}{ll}
A^{\circ} \cap B^{\circ} & A^{\circ} \cap \partial B \\
\partial A \cap B^{\circ} & \partial A \cap \partial B
\end{array}\right]
$$

Where $\quad A^{\circ}=$ the interior of region A

$\partial A=$ the boundary of region $\mathrm{A}$

$B^{\circ}=$ the interior of region $\mathrm{B}$

$\partial B=$ the boundary of region $\mathrm{B}$

The eight relations are showed as follow: $\operatorname{disjoint}(\mathrm{A}, \mathrm{B})=\left[\begin{array}{ll}0 & 0 ; 0\end{array}\right.$ $0], \operatorname{meet}(A, B)=\left[\begin{array}{lll}0 & 0 ; 0 & 1\end{array}\right], \operatorname{overlap}(A, B)=\left[\begin{array}{lll}1 & 1 ; 1 & 1\end{array}\right], \operatorname{cover}(A, B)$ $=\left[\begin{array}{lll}1 & 1 ; 0 & 1\end{array}\right]$, contain $(\mathrm{A}, \mathrm{B})=\left[\begin{array}{lll}1 & 1 ; 0 & 1\end{array}\right]$, coveredBy $(\mathrm{A}, \mathrm{B})=\left[\begin{array}{ll}1 & 0 ; 1\end{array}\right.$ $1]$, containedBy $=\left[\begin{array}{lll}1 & 0 ; 1 & 0\end{array}\right]$, equal $(A, B)=\left[\begin{array}{llll}1 & 0 ; & 0 & 1\end{array}\right]$.

In "9-Intersection" Model (9IM) (Egenhofer, 1990a;Egenhofer, 1993), for definition of topological relations, in addition to interiors and boundaries of the regions, the exteriors are also considered. The "9-Intersection" model easily extends the "4Intersection" to nine elements using a $3 * 3$ matrix.

$$
T(A, B)=\left[\begin{array}{lll}
A^{\circ} \cap B^{\circ} & A^{\circ} \cap \partial B & A^{\circ} \cap \mathrm{B}^{e} \\
\partial A \cap B^{\circ} & \partial A \cap \partial B & \partial A \cap \mathrm{B}^{e} \\
A^{e} \cap B^{\circ} & A^{e} \cap \partial B & A^{e} \cap \mathrm{B}^{e}
\end{array}\right]
$$

Where $\quad A^{c}=$ the interior of region A

$\partial A=$ the boundary of region $\mathrm{A}$

$A^{e}=$ the exteriors of region $\mathrm{A}$

$B^{\circ}=$ the interior of region $\mathrm{B}$

$\partial B=$ the boundary of region $\mathrm{B}$

$B^{e}=$ the exteriors of region $\mathrm{B}$

Even though the exterior of objects adds more expressiveness to the topological relations, no more relations between regionregion are distinguished in "9-Intersection" model (Chen, 2001). The topological relations of "9-Intersection" are defined for eight relations as follows (Clementini, 1994): disjoint(A,B) $=\left[\begin{array}{lllllllll}0 & \delta & \delta ; & \delta & 0 & \delta ; & \delta & \delta & \delta\end{array}\right], \operatorname{meet}(\mathrm{A}, \mathrm{B})=\left[\begin{array}{llllll}0 & \delta & \delta ; & \delta & 1 & \delta ; \delta\end{array}\right.$ $\left.\begin{array}{ll}\delta & \delta\end{array}\right]$, overlap $(\mathrm{A}, \mathrm{B})=\left[\begin{array}{cccccccc}\delta & 1 & \delta ; 1 & \delta & \delta ; \delta & \delta & \delta\end{array}\right], \operatorname{cover}(\mathrm{A}, \mathrm{B})$

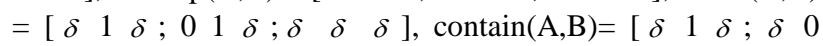
$\left.\begin{array}{llll}\delta & \delta & \delta & \delta\end{array}\right]$, coveredBy(A,B) $=\left[\begin{array}{llllllll}\delta & 0 & \delta ; 1 & 1 & \delta ; \delta & \delta & \delta\end{array}\right]$, containedBy $=\left[\begin{array}{llllllll}\delta & \delta & \delta ; 1 & 0 & \delta ; & \delta & \delta & \delta\end{array}\right]$, equal(A,B) $=\left[\begin{array}{ll}\delta \\ \delta\end{array}\right.$ $\left.\begin{array}{llllllll}\delta & \delta & 0 & \delta & 0 ; & \delta & \delta & \delta\end{array}\right]$. Here 0 and 1 represent the empty and non-empty, respectively. Each $\delta$ indicates a value that we don't care. The 4IM and 9IM are easy to be implemented in practical applications. But they have limitations to determine the more expressively relations between parts of a 3D complex object.

2.2.3 The Dimensionally Extended Model: For the purpose of describing detailed topological relations, the dimensionally extended method was presented by Clementini et al. (Clementini, 1993). In this method, values $-1,0,1$ and 2 are used to qualify the intersection between two regions. If there is no intersection, -1 is used to indicate null set. 0 implies that intersection result contains, at least, one point and no lines or areas. Similarly, 1 indicates that the intersection contains, at least, a line and no area. Finally, 2 indicates that the intersection contains at least an area. Based on these definitions, the dimension of intersection is taken into account in the topological relations. The five topological relations, including touch, in, cross, overlap and disjoint, are defined and analysed. These relations are used to define the topological relations among point, line, and area. These relations are proved to be mutually exclusive because they meet the criteria of Jointly Exhaustive and Pairwise Disjoint (JEPD). A decision tree is provided to discriminate topological relationships with the aid of dimension definition. Additionally, Multi-level topological relations are presented based on 4-Intersection model. The intersection and difference model replaces the original intersection model for reducing the computation complexity of spatial operation between regions. Moreover, the definitions of topological complexity and topological distance are introduced to classify the eight relations. Five topological invariants are applied to decide further the detailed level of topological relations hierarchically. This method can determine more detailed topological relations between two region based on 
topological invariants (Deng, 2007).

The Dimensionally Extended 9-Intersection Model (DE-9IM) (Strobl, 2008) is a full descriptive assertion about two spatial objects in $R^{2}$. The "9-Intersection" model belongs to binary classification. The values of elements in "9-Intersection" model can be either empty or non-empty. However, the corresponding elements in DE-9IM become the dimension operation of those elements in the "9-Intersection" model.

$T(A, B)=\left[\begin{array}{lll}\operatorname{dim}\left(A^{\circ} \cap B^{\circ}\right) & \operatorname{dim}\left(A^{\circ} \cap \partial B\right) & \operatorname{dim}\left(A^{\circ} \cap B^{e}\right) \\ \operatorname{dim}\left(\partial A \cap B^{\circ}\right) & \operatorname{dim}(\partial A \cap \partial B) & \operatorname{dim}\left(\partial A \cap B^{e}\right) \\ \operatorname{dim}\left(A^{e} \cap B^{\circ}\right) & \operatorname{dim}\left(A^{e} \cap \partial B\right) & \operatorname{dim}\left(A^{e} \cap B^{e}\right)\end{array}\right]$

Where $\quad A^{\circ}=$ the interior of region $\mathrm{A}$

$\partial A=$ the boundary of region $\mathrm{A}$

$A^{e}=$ the exteriors of region $\mathrm{A}$

$B^{\circ}=$ the interior of region $\mathrm{B}$

$\partial B=$ the boundary of region $\mathrm{B}$

$B^{e}=$ the exteriors of region $\mathrm{B}$

$\operatorname{dim}()=$ dimension operator

Here $\operatorname{dim}(s)=\max \left\{\operatorname{dim}\left(s_{1}\right), \operatorname{dim}\left(s_{2}\right), \ldots, \operatorname{dim}\left(s_{n}\right)\right\}$, and $s_{i}$ is the spatial set of intersection of the interior, boundary and exterior of region A and B. So the possible dimension values are in the set of $\{-1,0,1,2\}$. -1 means empty set, 0 for points, 1 for lines and 2 for areas. But for querying of topological relations, the $3^{*} 3$ matrix are formatted as a string code. The DE-9IM code is an accepted standardized format in the OGC standards. The DE9IM have been implemented in PostGIS for data analysis (Boundless, 2014). More importantly, it can transform geometric information into semantical descriptions of topological relations.

2.2.4 RCC in 3D Space: Among all the 512 possible relations in 9IM, eight relations are easily recognizable in $R^{2}$. Similarly, eight relations can apply to 3D objects in $R^{3}$ (Zlatanova, 2004). Because RCC may define regions as continuous space representation, Generalized 2D Region Connection Calculus (GRCC) (Li, 2004) extends RCC for both infinite real space and discrete space for the purpose of analysing topological relations between regions in discrete space, such as regions extract from images and point clouds. Thus, RCC-3D (Albath, 2010b) extends the spatial reasoning in $R^{3}$ based on GRCC, and it introduces new relations by adding the relations of objects projected in principle planes perpendicularly in $\mathrm{R}^{3}$ (ex. objects projected to planes formed by xy-axes, yz-axes and zx-axes). The combination of five predicates and a converse predicate can uniquely identify 13 RCC-3D relations between a pair of 3D objects or multiple objects in the case of no a priori knowledge about the underlying relations(Albath, 2010a). VRCC-3D+ (Sabharwal, 2011) used RCC-3D and depth parameter to distinguish nonocclusion, partial occlusion and complete occlusion relations in $R^{3}$, which relies on the viewpoints and the projection planes. Essentially, these methods transform the topological relations into 2D plane to determine relations in $R^{3}$. Meanwhile, the definitions of topological relations models are derived from RCC-8 in $R^{2}$.however, these models cannot be applied to determine topological relations among object components in the B-Rep 3D models.

In conclusion, RCC-8 is the used to define topological relations in $R^{2}$. It is also used for analysing the topological relations in
$R^{3}$. For the topological relations among components of objects represented by the B-Rep model, the extended DE-9IM is more effective. This model helps to describe the topological relations among components of a complex object by integrating information from parametric approach.

\section{TOPOLOGICAL RELATIONSHIPS AMONG 3D PLANAR REGIONS FORMING A COMPLEX 3D OBJECT}

\subsection{Definition of Topological Model for Planar Region}

For determination of topological relations between planar regions in $3 \mathrm{D}$ space, their boundary and interior of planar regions are critical. The geometric representation of a plane in $R^{3}$ is formally defined by $\mathrm{Ax}+B y+C z+D=0$. But this equation defines a plane without any boundary. Thus, we need not only define a planar region by a plane equation, but also we need to determine its boundaries, and its interior.

Topological relations between planar regions in $R^{3}$ are firstly dependent on the spatial relations between two planes (SRp) in which planar regions locate. Then topological relations of planar regions (TRr) can be determined based on SRp. The set of SRp is \{parallel, coplanar, intersecting $\}$. TRr still are defined based on the eight topological relations defined in 4IM. If the value of SRp is parallel, two planar regions must be disjoint. If the value of SRp is coplanar, then the relations between 3D planar regions become relations between regions in $2 \mathrm{D}$ space. The intersecting case of SRp results in more detailed topological relations between planar regions in $R^{3}$.

When SRp is the intersecting case, according to the definitions of the calculus-based model, "Disjoint" is the case that there is no common part between two planar regions. "Meet" is decided when there is and only is a common part of the boundaries of two planar regions in the intersection line. Except disjoint and meet, intersect relation covers all other remaining relations. The main topological relations for the intersecting case of SRp can be classified as disjoint, meet and intersect cases. The topological relations between planar regions can be divided into the relations between intersecting line of two planes and two planar regions because intersecting line is the only possible connection between two planar regions.

Based on the spatial relations of planes, the topological relations of two planar regions are closely related to the relations between intersection line of two planes and each planar region. Therefore, intersection line, the boundaries and interiors of two planar regions are used to define topological relations between planar regions in a $3 * 3$ matrix as in DE-9IM as follows:

$$
T_{p}(A, B)=\left[\begin{array}{lll}
\operatorname{dim}\left(A^{\circ} \cap B^{\circ}\right) & \operatorname{dim}\left(A^{\circ} \cap \partial B\right) & \operatorname{dim}\left(A^{\circ} \cap I l_{B}\right) \\
\operatorname{dim}\left(\partial A \cap B^{\circ}\right) & \operatorname{dim}(\partial A \cap \partial B) & \operatorname{dim}\left(\partial A \cap I l_{B}\right) \\
\operatorname{dim}\left(I l_{A} \cap B^{\circ}\right) & \operatorname{dim}\left(I l_{A} \cap \partial B\right) & \operatorname{dim}\left(I l_{A} \cap I l_{B}\right)
\end{array}\right]
$$

Where $\quad \mathrm{A}^{\circ}=$ indicates the interior of region $\mathrm{A}$

$\partial \mathrm{A}=$ the boundary of $\mathrm{A}$

$\mathrm{B}^{\circ}=$ the interior of region $\mathrm{B}$

$\partial B=$ the boundary of region $\mathrm{B}$

$I l=$ intersection of two planes containing two planar

regions. Here $I l_{\mathrm{A}}$ and $I l_{\mathrm{B}}$ share the same line equation.

$\operatorname{dim}()=\operatorname{dimension}$ operator 
Because the value of $\operatorname{dim}\left(I l_{A} \cap I l_{B}\right)$ always is 1 , it cannot provide more details to describe topological relations. However, the intersection primitives of $I l$ and a planar region could be points or line segments. For providing detailed descriptions of topological relations between these primitives, the $\operatorname{dim}\left(I l_{A} \cap I l_{B}\right)$ is replaced by $\zeta$. The $\zeta$ indicates topological relations of two parts of intersection primitives (points and lines) constituted by the intersecting line and two planar regions individually. Thus, the original matrix is represented as follow:

$T_{p}^{\prime}(A, B)=\left[\begin{array}{ccc}\operatorname{dim}\left(A^{\circ} \cap B^{\circ}\right) & \operatorname{dim}\left(A^{\circ} \cap \partial B\right) & \operatorname{dim}\left(A^{\circ} \cap I l_{B}\right) \\ \operatorname{dim}\left(\partial A \cap B^{\circ}\right) & \operatorname{dim}(\partial A \cap \partial B) & \operatorname{dim}\left(\partial A \cap I l_{B}\right) \\ \operatorname{dim}\left(I l_{A} \cap B^{\circ}\right) & \operatorname{dim}\left(I l_{A} \cap \partial B\right) & \zeta\end{array}\right]$

Topological relations between intersection primitives lying in the intersection line are comprised of the relations of pointpoint, point-line, line-line in the same line equation, so it contains disjoint, meet, overlap, covers, contains, coveredBy, containedBy and equal as well (as shown in Table 2 ). Additionally, $\zeta$ is represented by a list of string, including the description of topological relations between primitives in intersection line, $I l_{A}^{r l}$ and it geometric type, $I l_{B}^{r l}$ and its type, the common part of $I l_{A}^{r l}$ and $I l_{B}^{r l}$, here $I l_{A}^{r l}$ indicates the common parts of region $\mathrm{A}$ and the intersection line, and $I l_{B}^{r l}$ means the common parts of region B and the intersection line. Therefore, $\zeta$ is designed to discriminate the detailed topological relations on the basis of other eight elements of the matrix.

\begin{tabular}{|l|l|}
\hline Type of relations & Graphical representation \\
\hline $\begin{array}{l}\text { Point-point } \\
\text { relations }\end{array}$ & \\
\hline $\begin{array}{l}\text { Line segment-point } \\
\text { relations }\end{array}$ & - disjoint \\
\hline $\begin{array}{l}\text { Line segment-line } \\
\text { segment relations }\end{array}$ & - contain \\
& - \\
& - \\
& -
\end{tabular}

Table 2. Basic topological relations between primitives in the intersection line of two planes (red primitives are the intersections between planar region $\mathrm{A}$ and the intersecting line, and yellow parts are the intersections between planar region B and the intersecting line)

As shown in Figure 1, for the case of disjoint between line segment and line segment, $\zeta=$ [disjoint, $<P_{1 A} P_{2 A}$, line segment $>,\left\langle P_{1 \mathrm{~B}} P_{2 \mathrm{~B}}\right.$, line segment $\left.>, \varnothing\right]$.

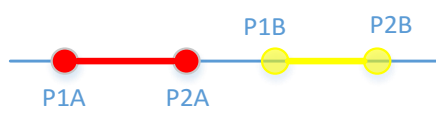

Figure 1. Disjoint case between line segments resulted from the intersection of two planar regions and intersecting line of two planes
Similarly, for the case of overlap, $\zeta=$ [overlap, $\left\langle P_{1 A} P_{2 A}\right.$, line segment $>,\left\langle P_{1 \mathrm{~B}} P_{2 \mathrm{~B}}\right.$, line segment $>,\left\langle P_{1 \mathrm{~B}} P_{2 \mathrm{~A}}\right.$, line segment $>$ ]. For meet relation we have $\zeta=$ [meet, $\left\langle P_{1 A} P_{2 A}\right.$, line segment $>$, $<P_{1 \mathrm{~B}} P_{2 \mathrm{~B}}$, line segment $>,<P_{2 \mathrm{~A}}$, point $>$ ].

However, for those regions with holes, the $\zeta$ is composed of a set of relations that is represented by a list of $\zeta_{i}$. For example, in Figure 2, there is a hole in region B. for this case, $\zeta=[$ [overlap, $\left\langle P_{1 \mathrm{~A}} P_{2 \mathrm{~A}}\right.$, line segment $>,\left\langle P_{1 \mathrm{~B}} P_{2 \mathrm{~B}}\right.$, line segment $>$, $\left\langle P_{1 \mathrm{~A}} P_{2 \mathrm{~B}}\right.$, line segment $>$ ]; [overlap, $\left\langle P_{1 A} P_{2 A}\right.$, line segment $>$, $\left\langle P_{3 \mathrm{~B}} P_{4 \mathrm{~B}}\right.$, line segment $\rangle,\left\langle P_{3 \mathrm{~B}} P_{2 \mathrm{~A}}\right.$, line segment $\left.\left.>\right]\right]$. Therefore, the last element $\zeta$ in the matrix is an effective complementary for the description of topological relations of two planar regions. In summary, a detailed representation of topological relations of two planar regions consists of SRp and topological matrix with $\zeta$.

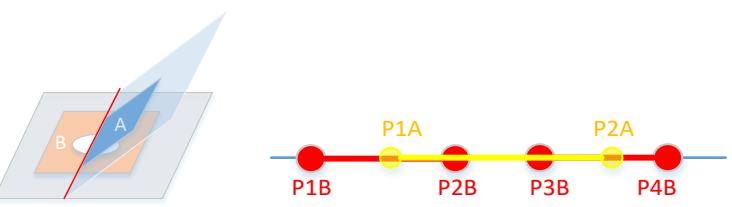

Figure 2. Topological relations of two planar regions with holes

\subsection{Definition of Topological Relations between Planar Regions}

In the following section, we present some examples for disjoint, meet and intersect relations and their representations. One should note again that the intersection relation contains different cases of the relations between two planar regions as presented in previous section.

3.2.1 Disjoint: According to the definition of disjoint relation in $9 \mathrm{IM}$, disjoint $(\mathrm{A}, \mathrm{B})=\left[-1-1^{*} ;-1-1^{*} ; * * \zeta\right]$ is used to decide disjoint relation between planar regions in $R^{3}$. However, there are several cases for the disjoint relation between two planar regions in $R^{3}$. For example, in Figure 3(3) and 3(6), the relations between $\mathrm{A}$ and $\mathrm{B}$ are represented with the same element $\zeta$. But combining other eight elements in the matrix, $\zeta$ can be used as a key element to differentiate those cases in $R^{3}$.
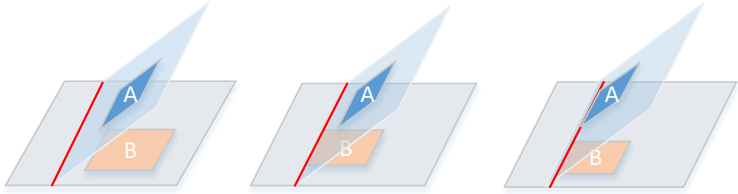

$[-1-1-1 ;-1-1-1 ;-1-1 \zeta][-1-1-1 ;-1-1-1 ;-11 \zeta][-1-1-1 ;-1-11 ;-11 \zeta]$
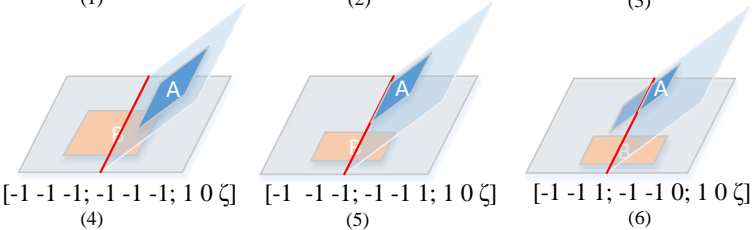

Figure 3. Disjoint relations of two planar regions

3.2.2 Meet: For a meet relation, there are six common cases that we can distinguish. In Figure 4(1), $\zeta$ is the case of meet relation. In (2), (3) and (4), they have the same previous eight 
elements in the matrix, but $\zeta$ is various. They are overlap, equal and contain relations respectively. But in (1), (5) and (6), they have the same $\zeta$.
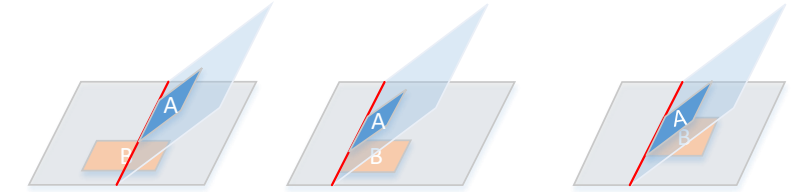

$[-1-1-1 ;-1$

(2)

(3)

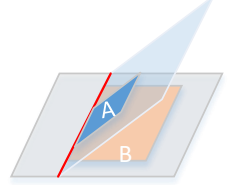

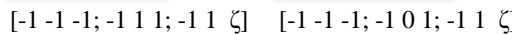
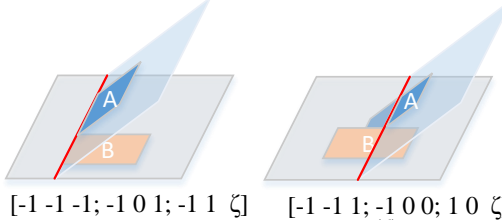

Figure 4. Meet relations of two planar regions

3.2.3 Intersect: The following six cases are common relations for the intersection relations between two planar regions. In Figure 5(1) and (4), two $\zeta$ representations are the overlap case. For (2) and (5), they are the equal case. In the same way, $\zeta$ in (3) and (6) are the contain case.

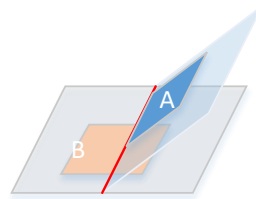

$[-1-1-1 ; 101 ; 100 \zeta]$ (1)

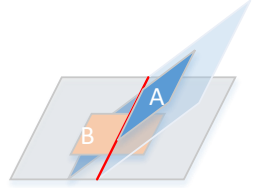

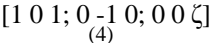

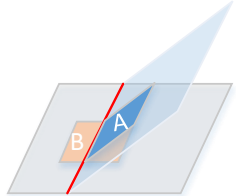

$[-1-1-1 ; 101 ; 10 \%$ $(2$

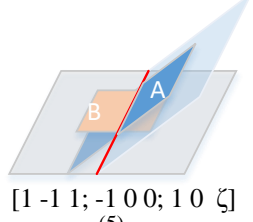

(5)

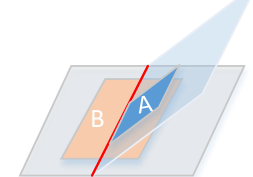

[-1 - $1-1 ; 1-1 \quad 1 ; 10 \%]$

(3)

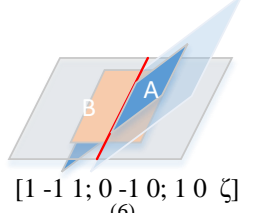

(6)
Figure 5. Intersect relations of two planar regions

\subsection{The Discriminant of Topological Relations between Planar Regions}

The discriminant of topological relations between planar regions needs four steps:

Step 1: Compute parameters of plane equations for two planar regions in a same Cartesian coordinate system in $R^{3}$;

Step 2: Compute the spatial relations of planes (SRp);

Step 3: Decide the topological relations between planar regions based on SRp;

a) If SRp is parallel, topological relations of planar regions (TRr) is disjoint;

b) If $\mathrm{SRp}$ is coplanar, $\mathrm{TRr}$ is the case of topological relations between regions in $2 \mathrm{D}$ space;

c) If SRp is intersecting, firstly, calculate the intersecting line equation of two planes and decide the common parts of planar regions and intersecting line; then, compute the elements of topological matrix, including the last element $\zeta$ as described in the previous section;

Step 4: Provide semantic descriptions topological relations after geometric computation and analysis of matrix;
The first three steps are done by geometric computation. In the third step (c), the topological relations can be defined by a $2 * 2$ submatrix $\quad T_{2 \times 2}(A, B)=\left[\begin{array}{ll}\operatorname{dim}\left(A^{\circ} \cap B^{\circ}\right) & \operatorname{dim}\left(A^{\circ} \cap \partial B\right) \\ \operatorname{dim}\left(\partial A \cap B^{\circ}\right) & \operatorname{dim}(\partial A \cap \partial B)\end{array}\right]$ in the upper left of $T_{p}^{\prime}(A, B)$. For the disjoint case, $T_{2 \times 2}(A, B)=[-1,-1$; $-1,-1]$, it is same as the case in $2 \mathrm{D}$ space. For the meet case, $T_{2 \times 2}(A, B)=[-1,-1 ;-1, *]$, here * could be 0 or 1 . According to the matrix $T_{p}{ }^{\prime}(A, B)$, the intersect case also can be decided by matching the elements from matrixes in Figure 5. Additionally, for each case, $\zeta$ can be defined and stored following the predefined formats in section 3.1. The detailed intersected information is described in $\zeta$. If there are more than one list $\zeta_{i}$ in $\zeta$, it indicates that there are holes that pass the intersecting line. The common part in $\zeta$ is the connection part of two planar regions. Therefore, the topological relations between two planar regions in $R^{3}$ are obtained through the geometric computation and the predefined matrix to describe topological relations and the connection of planar regions.

\subsection{Challenges for Extraction of Topological Relations between Planar Regions Obtained from Point Cloud}

Point clouds can be observed by Light Detection And Ranging (LiDAR) devices, including terrestrial and airborne LiDAR. In a point cloud, information are contained in high volume points. Each point has several attributes defining coordinate $(\mathrm{x}, \mathrm{y}, \mathrm{z})$, intensity, classification, number of returns and point source ID and so on. However, despite the high density of points from surfaces, for occlusion cases, there may be missing parts in scanned LiDAR data that lead to incomplete segmentation of objects components. This can affect the determination of boundaries of each component of 3D complex object. Each component can be represented as a planar region and be used for obtaining topological relations between those components. For example, a wall could be modelled as a rectangle planar region. However, the boundary extracted from point clouds is not a perfect rectangle. A concave polygon extracted from a component cannot be directly estimated as a rectangle because the boundary constituted by points of a concave polygon is difficult to be ensured to form line segments of rectangle perfectly. As shown in Figure 6(1), following the segmentation step, six segments are identified in the point cloud for defining the building walls. From the top view in Figure 6(3), these segments look to be connected together perfectly. However when the boundaries of those segments are extracted, their topological relations are not perfect. We can see several gaps between the blue part and yellow part (Figure 6(2) and 6(4)). These imperfections affect the extraction of final boundaries and the determination of topological relations between those segments. Besides, the boundaries quality also depends on the quality of the point cloud. Thus, boundaries of components cannot be directly modelled as some primitives. In sum, extracting topological relations among components of a complex 3D object obtained from 3D LiDAR point clouds is very complex. Because the planar regions are not perfectly embed in a plane as supposed in the previous sections. The boundaries of those regions are very irregular and composed of concave hulls of points composing the region. These may become more complex if we deal with occlusion presence in point clouds. 

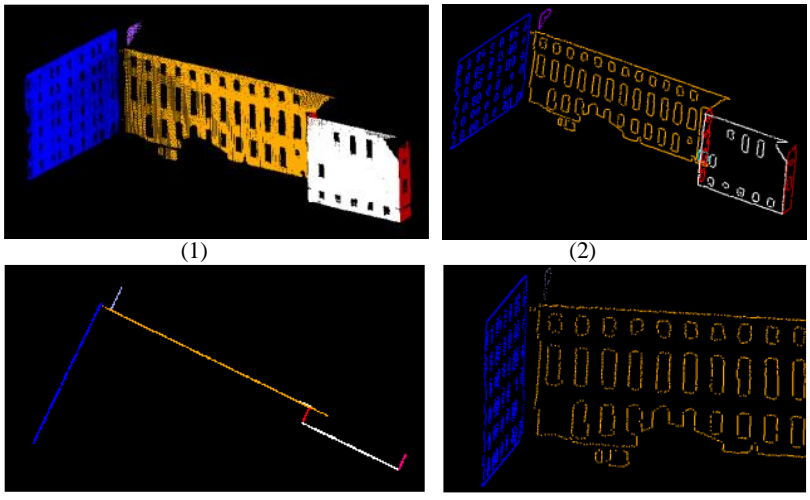

(3)

(4)

Figure 6. Examples of the components of a building and their boundaries obtained from a point cloud

\section{EXPERIMENTAL ANALYSIS}

In the automatic modelling from point clouds, the existing segmentation algorithms can detect planar components from point cloud because the model-based geometric detection algorithms are capable of detecting planes and acquiring parameter of planes from point clouds. For example, Random Sample Consensus can segment simple primitives, such as plane, sphere, cylinder and cone. After segmentation, in Figure 7(1), 16 pieces of planar components are extracted from a building. From the top view of the building, lines with different colours indicate different walls. In Figure 7(2), this building is displayed from another view. Five walls of this building are presented in Figure 7(3). Each segment has geometric properties after segmentation. For example, a segment can be represented by geometric parameters (A, B, C, D) for a plane equation. We use the equations of two planes in $3 \mathrm{D}$ space to determine the equation of their intersecting line (L). Consider that $a_{1} X+b_{1} Y+c_{1} Z+d_{1}=0$ and $a_{2} X+b_{2} Y+c_{2} Z+d_{2}=0$ to be two plane equations. The direction vector of the line $\mathrm{L}$ is orthogonal to the normal vectors of two planes $n_{1}=\left(a_{1}, b_{1}, c_{1}\right)$ and $n_{2}=\left(a_{2}, b_{2}, c_{2}\right)$. The direction vector is obtained by $\mathrm{s}=n_{1} \times n_{2}$. If $M\left(x_{1}, y_{1}, z_{1}\right)$ is a common point between the two plane then the line equation is defined as:

$$
\frac{X-x_{1}}{p}=\frac{Y-y_{1}}{q}=\frac{Z-z_{1}}{r}
$$

Where $p=b_{1} * c_{2}-b_{2} * c_{1}, q=c_{1} * a_{2}-c_{2} * a_{1}, r=a_{1} * b_{2}-a_{2} * b_{1}$

To determine the common parts of two planar regions and the intersecting line, the distance between points of planar regions and line is used to make a decision. The distance between a point $M_{0}\left(x_{0}, y_{0}, z_{0}\right)$ and the line $\mathrm{L}$ in $3 \mathrm{D}$ space can be computed by following equation:

$$
d^{2}=\left(x_{0}-x_{1}\right)^{2}+\left(y_{0}-y_{1}\right)^{2}+\left(z_{0}-z_{1}\right)^{2}-\frac{\left[p\left(x_{0}-x_{1}\right)+q\left(y_{0}-y_{1}\right)+r\left(z_{0}-z_{1}\right)\right]^{2}}{p^{2}+q^{2}+r^{2}}
$$

In order to compute the value of $\zeta$, we need to determine if both planar regions have interstation with line L. To do so, we define a distance threshold which is determined by the average distance between $\mathrm{M}$ and its K-nearest neighbours. Thus, each point in the planar regions has its distance threshold to judge whether it is on the line. By considering the K-nearest neighbours of $\mathrm{M}$, we make sure that the distance is determined based on the local density of points. Here $\mathrm{K}$ is defined by the density of point cloud. If the distance between point $M$ belonging to one of the regions and the line $\mathrm{L}$ is less than the threshold of this point, then we consider that this point is on the line L. Next, those points belonging to one of planar regions on the line $\mathrm{L}$ are combined to create line segments using the same distance threshold used in previous step. If the distance between point $\mathrm{M}$ and its nearest boundary point $\mathrm{N}$ on the line are in the distance threshold of $\mathrm{M}, \mathrm{M}$ and $\mathrm{N}$ are added to the same line segment. In the next step, $\mathrm{N}$ is the new starting point to search other points on the line. This action is repeated until all the points of a planar region are processed. In this way, the line segments formed by the common part of the intersection line $\mathrm{L}$ and the planar region are obtained. This process is also carried out for the second planer region in the same way. The points of planar regions have two classes: boundary and interior. Therefore, the line segments are easy to be identified as the intersecting part of line $\mathrm{L}$ and the boundary or the interior. Finally, the line segments belonging to two regions on the line $\mathrm{L}$ are used to determine $\zeta$ according to the steps presented in Section 3.1.

For distinguishing the boundary and interior of each segment, the concave hull of a planar segment is extracted from the point cloud, which is implemented by algorithms in Point Cloud Library (Rusu, 2011). As shown in Figure 7(4), the white planar region and blue one have the meet relation, similarly, the pink one has meet relation with the blue one. These relations are computed using the topology matrix following the steps presented in Section 3.3. Therefore, the topological relations between two neighbouring segments are obtained by the topology matrix and the last element $\zeta$.

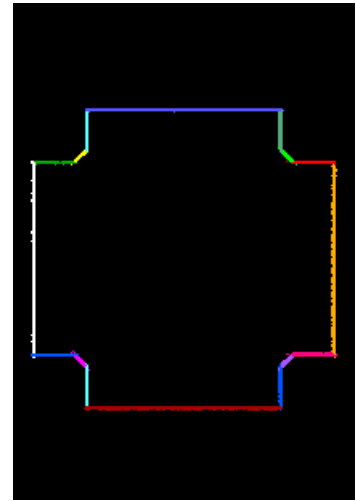

(1)

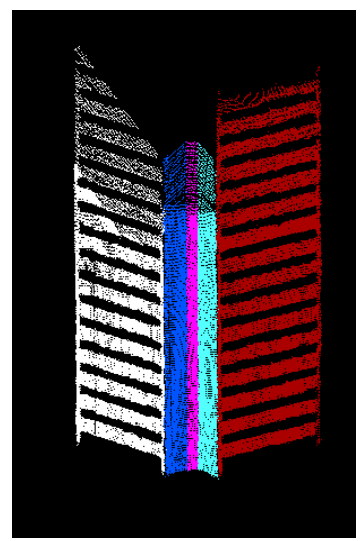

(3)

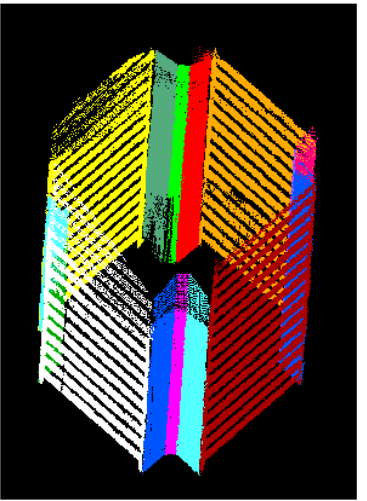

(2)

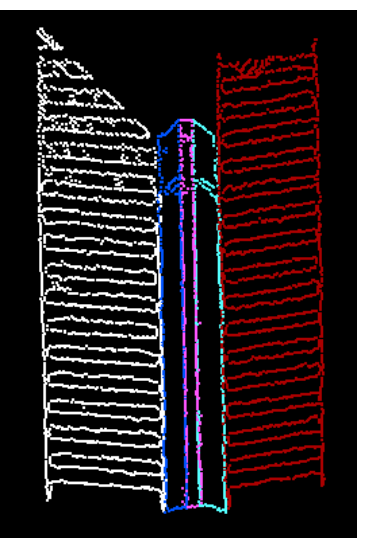

(4)
Figure 7. Results for planar regions segmented from point cloud

\section{CONCLUSION AND FUTURE WORK}

The topological relations between planar regions in $R^{3}$ are extended from Dimension Extended 9-Intersection models. The extended model is more expressive and allows better 
distinguishing and describing the topological relationships among planar regions. Furthermore, it can transform geometric information into topological relations between components of 3D complex objects based on basic geometric computations and the analysis of topology matrix. The proposed approach describes not only the topological relations, but also the details of the relation of the connection parts between planar regions. Moreover, the topological relations of planar regions with holes can be represented by the proposed approach. We have also analysed different challenges that we have when applying the proposed method for the determination of topological relations between two planar regions extracted from point clouds. Finally, the proposed topological models is applied to identify the topological relations between planar regions extracted from point cloud automatically. Future work will be focused on extending topological relations of planar regions to other geometric primitives. Also, the data structure for topological relations of objects components will be designed to realize spatial querying and analysis on complex 3D objects. Furthermore, we will explore the creation of complete B-Rep models based on fundamental topological relations in 3D complex models.

\section{ACKNOWLEDGEMENTS}

This research was made possible by an operating grant from Natural Sciences and Engineering Research Council of Canada (NSERC). The authors would like to gratefully acknowledge dataset provider for the experimentations presented in this paper.

\section{REFERENCES}

Albath, J.,2010a. Efficient Reasoning with RCC-3D. In: Knowledge Science, Engineering and Management, pp. 470481.

Albath, J.,2010b. RCC-3D: Qualitative Spatial Reasoning in $3 D$. In: Proceedings of the 23rd International Conference on Computer Applications in Industry and Engineering(CAINE 2010) Las Vegas, NV, Vol. pp. 74-79.

Boundless, 2014). Dimensionally Extended 9-Intersection model." Introduction to PostGIS", http://workshops.boundlessgeo.com/postgis-intro/de9im.html

Chen, J., 2001. A Voronoi-based 9-intersection model for spatial relations. International Journal of Geographical Information Science, 15(3),pp. 201-220.

Clementini, E.,1993. A Small Set of Formal Topological Relationships Suitable for End-User Interaction. In: Advances in Spatial Databases, pp. 277-295.

Clementini, E., 1994. Modelling topological spatial relations: Strategies for query processing. Computers \& Graphics, 18(6),pp. 815-822.

Cohn, A. G., 2008. Qualitative spatial representation and reasoning. Handbook of knowledge representation, 3pp. 551596.

Deng, M., 2007. Multi-level Topological Relations Between Spatial Regions Based Upon Topological Invariants. GeoInformatica, 11(2),pp. 239-267.
Egenhofer, M.,1989. A formal definition of binary topological relationships. In: Foundations of Data Organization and Algorithms, pp. 457-472.

Egenhofer, M.,1993. Topological relations between regions in $\rho 2$ and $\mathbb{Z} 2$. In: Advances in Spatial Databases, pp. 316-336.

Egenhofer, M. J., 1991. Point-set topological spatial relations. International Journal of Geographical Information System, 5(2),pp. 161-174.

Egenhofer, M. J.,1990a. Categorizing binary topological relations between regions, lines, and points in geographic databases. In: Technical Report, Orono, pp. 94-122.

Egenhofer, M. J.,1990b. A mathematical framework for the definition of topological relationships. In: Fourth international symposium on spatial data handling, Vol. pp. 803-813.

Foley, J. D.,1996. 12.7 Constructive Solid Geometry. In: Computer graphics (2nd ed. in $\mathrm{C}$ ): principles and practice, pp. $557-558$.

JARROUSH, J.,2004. Constructive Solid Geometry as the Basis of $3 D$ Future Cadastre. In: FIG working Week, Athens, Greece, pp. 1-14.

Koussa, C.,2009. A simplified geometric and topological modeling of $3 D$ buildings: combination of surface-based and solid-based representations. In: ASPRS 2009 annual Conference: Reflection of the past, vision of the future, Maryland, Vol. pp. 1-13.

Leopold, J. L., 2015. Spatial relations between 3D objects: The association between natural language, topology, and metrics. Journal of Visual Languages \& Computing, 27pp. 29-37.

Li, S., 2004. Generalized Region Connection Calculus. Artificial Intelligence, 160(1-2),pp. 1-34.

Mark, D. M., 1994. Modeling spatial relations between lines and regions: combining formal mathematical models and human subjects testing. Cartography and geographic information systems, 21(4),pp. 195-212.

Pigot, S.,1991. Topological models for 3d spatial information systems. In: AUTOCARTO-CONFERENCE, Vol. 6 pp. 368392.

Randell, D. A.,1992. A Spatial Logic based on Regions and Connection. In: Proceeding 3rd International Comference on Knowledge Representation and Reasoning, Morgan, Kaufmann, Vol. pp. 1-12.

Rusu, R. B.,2011. 3D is here: Point Cloud Library (PCL). In: IEEE International Conference on Robotics and Automation (ICRA), Shanghai, China, Vol. pp. 1-4.

Sabharwal, C. L.,2011. A More Expressive 3D Region Connection Calculus. In: DMS, Vol. pp. 307-311.

Strobl, C.,2008. Dimensionally Extended Nine - Intersection Model (DE-9IM). In: Encyclopedia of GIS, pp. 240-245.

Zlatanova, S., 2004. Topological models and frameworks for 3D spatial objects. Computers \& Geosciences, 30(4),pp. 419428. 